\title{
DERIVATI FENAZINICI COME POTENZIALI AGENTI PER IL TRATTAMENTO DELLE INFEZIONI SOSTENUTE DA M.TUBERCULOSIS RESISTENTI E MULTIRESISTENTI
}

Saddi M. a, Palchykovska L.I. ', Borgna R. , Kostina V.G. Chisu L. ${ }^{a}$, Soru A. ${ }^{a}$, Shestakova T.S. ${ }^{b}$, Magnapane S. ${ }^{a}$, Alexeeva I.V. ${ }^{b}$, Saddi B. ${ }^{c}$, Shved A.D. ${ }^{b}$, De Logu A.

aDipartimento di Scienze e Tecnologie Biomediche, Sezione di Microbiologia Medica, Università di Cagliari, Viale Sant'lgnazio 38, 09123 Cagliari

bepartment of Molecular Virology, Institute

of Molecular Biology and Genetics,

Nationl Academy of Sciences of Ucraine,

150 Zabolotnogo Str., Kiev, Ucraine

Laboratorio di Analisi, Ospedale S.S. Trinità, Cagliari

Negli ultimi quindici anni si è verificato un aumento dell'incidenza della tubercolosi che rappresenta, tra le malattie infettive, la causa predominante di morte nell'uomo.

Nonostante la validità della chemioterapia a breve corso DOTS, Mycobacterium tuberculosis continua a mietere più vittime di qualsiasi altro patogeno come unico responsabile di malattia.

La selezione di ceppi capaci di eludere gli antibiotici attualmente in uso rende una nuova epidemia di tubercolosi un'eventualità da non sottovalutare.

Risulta per questo necessario individuare nuovi farmaci antitubercolari. Abbiamo valutato l'attività in vitro di una serie di derivati fenazinici nei confronti del ceppo di riferimento virulento M. tuberculosis H37Rv ATCC 27294 e sui ceppi di collezione (ATCC) con diversi patterns di resistenza, isolati clinici resistenti e MDR. È stata inoltre determinata l'attività in linee cellulari di macrofagi J774 infettati con M. tuberculosis.

Il confronto tra i valori di MIC ottenuti sul ceppo di riferimento virulento per i derivati fenazinici più attivi e quelli ottenuti per rifampicina (RMP), ha evidenziato che alcune delle molecole più attive mostrano valori di MIC inferiori o paragonabili ad essa.

Tutti i derivati fenazinici saggiati su ceppi di collezione resistenti, presentano valori di MIC notevolmente inferiori a quelli determinati per isoniazide (INH) e RMP nei confronti dei ceppi rispettivamente resistenti. Sugli isolati clinici INHresistenti abbiamo constatato che i derivati più potenti sono più attivi dell'etambutolo e di INH.

Un aspetto particolarmente interessante che emerge dai risultati ottenuti è rappresentato dal fatto che i ceppi, sia di collezione che di provenienza clinica, con caratteristiche di resistenza a RMP, che agisce, come i derivati studiati, mediante inibizione della RNA polimerasi, si dimostrano ugualmente sensibili all'azione dei derivati fenazinici. Queste considerazioni possono assumere un significato di grande rilevanza per un eventuale impiego clinico di tali derivati. 